\title{
PODERES TRADICIONALES, EMERGENTES Y RE-EMERGENTES: RELACIONES AMBIGUAS, PERO PRAGMÁTICAS
}

\author{
Clatrisa Giaccaglia
}

UNA REVISIÓN de lo sucedido con los poderes ${ }^{1}$ emergentes durante la década y media ya transcurrida de este nuevo siglo deja al descubierto que gran parte de los análisis -periodísticos, financieros e incluso académicos- se han elaborado sobre reflexiones rimbombantes que oscilaron desde una exageración sobre las potencialidades de las naciones emergentes, y sus consiguientes repercusiones para el orden en su conjunto, ${ }^{2}$ hasta pronósticos -absolutamente desfavorables sobre el futuro de estos poderes, echando por tierra toda posibilidad de incorporación de estas potencias a la estructura de gobernanza mundial. ${ }^{3}$

${ }^{1}$ Para los fines de este trabajo, y en función de lo estipulado por el Diccionario de la Real Academia Española, "poder" y "potencia" son utilizados indistintamente como sinónimos.

${ }^{2}$ Véanse, por ejemplo, Jim O’Neill, "Building Better Global Economic BRIcs", GS Global Economics, Paper 66, octubre de 2001, en http://www.goldmansachs. com/our-thinking/archive/archive-pdfs/build-better-brics.pdf; "Potencias emergentes: China, India, Brasil y Sudáfrica”, Vanguardia Dossier, núm. 12, 2004; The Boston Consulting Group, "The New Global Challenges. How 100 Top Companies from Rapidly Developing Economies are Changing the World”, BCG Report, Boston, 2006; "The New Titans", The Economist, 14 de septiembre de 2006; Francis Kornegay y Narnia Bohler Muller, Laying the brics of a New Global Order: From Yekaterinburg 2009 to Thekwini 2013, Sudáfrica, Africa Institute of South Africa, 2013, entre otros.

${ }^{3}$ Véanse Ruchir Sharma, "The broken BRICs", Foreign Affairs, noviembre-diciembre de 2012, en https://www.foreignaffairs.com/articles/brazil/2012-10-22/ 
La mayoría de estas observaciones se realizó tomando en consideración casi exclusivamente variables económicas (niveles de PBI, en especial) las cuales -sin desmerecer su importancia- resultan ser un indicador volátil e insuficiente para un análisis integral. En efecto, las economías emergentes se consolidaron, desde comienzos del siglo xxi, como las zonas más dinámicas de crecimiento del mundo. Esta aceleración se debió al ritmo de crecimiento de China que se expandió a una tasa promedio superior al $10 \%$ al año, e incluso llegó a crecer al 14\% en 2007. India promedió un crecimiento de $8 \%$, Rusia de $5 \%$ y Brasil y Sudáfrica alrededor de $4 \% .^{4}$

Como contrapartida, hoy en día, la debilidad del consumo global, el derrumbe de los precios de las materias primas y un conjunto de problemas económicos y políticos han hecho que dos miembros de los BRICs, Rusia y Brasil, caigan en recesión. La economía china, por su parte, se desaceleró. Esto a su vez ha desatado una fuga de capitales en los mercados emergentes. Sin embargo, India se ha diferenciado del resto, convirtiéndose en la economía de mayor progreso. ${ }^{5}$ Ahora bien, ¿hasta qué punto los vaivenes en las estadísticas nacionales influyen en la consideración o no de ciertas potencias susceptibles de generar reglas en el orden internacional? Se considera que el crecimiento nacional

broken-brics; "The great deceleration", The Economist, 27 de julio de 2013; Harsh V. Pant, "The BRIcs fallacy", The Washington Quarterly, vol. 36, núm. 3, 2013, pp. 91-105; Ian Talley "Los BRIcs pasan del brillo a las sombras", The Wall Street Journal Americas, reproducido en el diario La Nación, 20 de enero de 2016, en http://www.lanacion. com.ar/1863647-los-brics-pasan-del-brillo-a-las-sombras, entre otros.

${ }^{4}$ Datos del Fmi y del Banco Mundial en Luis Orgaz, Luis Molina y Carmen Carrasco, "El creciente peso de las economías emergentes en la economía y gobernanza mundiales. Los países BRIC", Documentos Ocasionales, núm. 1101, Banco de España, 28 de marzo de 2011.

${ }^{5}$ Con la llegada del gobierno de Narendra Modi se logró revertir la tendencia económica descendiente al crecer en un promedio de 7,5\% anual en 2014 y 2015. Véase “¿Cómo logró India convertirse en la nueva China de la economía mundial?, BBC Mundo, 9 de febrero de 2016, en http://www.bbc.com/mundo/ noticias/2016/02/160208_economia_india_nueva_china_lf 
de cada uno de los agentes estatales es uno de los factores incidentes para conformación de un determinado orden internacional. ${ }^{6}$ Con todo, no es el único ni el decisivo.

La cuestión de la jerarquización de los estados en el sistema internacional constituye una de las preocupaciones centrales de las Relaciones Internacionales como disciplina, en especial para el realismo en cuanto que tradición teórica dominante. El análisis de esta cuestión lleva inevitablemente a repensar sobre la estructura y la dinámica que presenta el sistema internacional en su conjunto abriendo un abanico de interrogantes respecto a los modos en que los diferentes actores estatales se organizan y comportan bajo una determinada configuración mundial. En este sentido, cabe preguntarse: ¿Quiénes son los poderes emergentes? ¿Qué papel están desempeñando, a partir del nuevo siglo, en los diversos ámbitos de negociación multilateral? ¿Cómo se han desarrollado los vínculos entre los grandes poderes tradicionales y los nuevos poderes en ascenso?

En el presente trabajo se reflexiona sobre el papel de los poderes emergentes, haciendo especial hincapié en los niveles de actividad diplomática y negociadora que estas potencias vienen desarrollando en diversas esferas de deliberación mundial. De este modo, se pretende ampliar la mirada, desde una perspectiva sólo basada en indicadores económicos nacionales, para incrementar el número de variables por considerar.

Desde una perspectiva realista, se parte de la premisa teórica de que todo orden internacional es moldeado por los grandes poderes ${ }^{7}$

${ }^{6}$ Se sigue la definición esgrimida por Robert Cox ("Multilateralism and World Order”, Review of International Studies, vol. 18, núm. 2, 1992, p. 161) para entender el "orden internacional": "configuración específica de poder en un momento histórico dado".

${ }^{7}$ Durante el llamado Concierto europeo, el concepto de gran potencia "servía para definir a aquellos estados con recursos (más o menos equivalentes) de todo tipo, incluido el militar, que creaban las reglas del juego gracias a su participación en las guerras y en las grandes conferencias diplomáticas”. Posteriormente, durante la Guerra Fría, las superpotencias -Estados Unidos y la Unión Soviética- fueron las que desempeñaron en el sistema bipolar un papel equivalente al de las grandes potencias europeas del Congreso de Viena de 1815. Por 
del momento. ${ }^{8}$ En este sentido, la hipótesis de trabajo aquí propuesta sostiene que los poderes medios emergentes han desplegado, a partir del nuevo siglo, una crecientemente significativa presencia en los ámbitos multilaterales de discusión global. Este accionar ha implicado mayores niveles de negociación con las grandes potencias tradicionales en un proceso caracterizado por relaciones predominantemente pacíficas, pero con altos grados de informalidad y pragmatismo. Esta dinámica implica el ejercicio de una cooperación limitada en el tiempo y focalizada en asuntos específicos -ya sean comerciales, financieros, sociales o medioambientales, entre otros. De este modo, el orden internacional se está moldeando bajo una lógica de manejo colectivo de los asuntos mundiales, en la cual participan tanto potencias tradicionales como emergentes y re-emergentes.

consiguiente, el término gran potencia se aplicó a un grupo de países con intereses mundiales. Este grupo incluyó, por un lado, a Francia, Gran Bretaña y China que, como miembros permanentes del Consejo de Seguridad de Naciones Unidas, comenzaron a desempeñar un papel jerárquicamente superior en el sistema internacional. Por otro, a la República Federal de Alemania y a Japón, que por sus recursos financieros y comerciales empezaron a tener un lugar prominente en el orden económico mundial (véase Esther Barbé, Relaciones internacionales, Madrid, Tecnos, 1995, pp. 147-150). La creación del G-7, en 1973, implicó la reunión de los países más industrializados del mundo en el entorno de la crisis económica internacional de los años setenta y frente a la necesidad de coordinación entre las potencias económicamente dominantes del sistema internacional. Finalmente, la disolución de la Unión Soviética significó, a inicios de la post-Guerra Fría, la existencia de una única superpotencia (Estados Unidos) acompañada por las restantes grandes potencias del G-7. En este trabajo, dicho conjunto de poderes se identifica bajo la categoría de potencias tradicionales.

${ }^{8}$ Según la Power transition theory, el sistema internacional es estable cuando existe una potencia dominante que define y hace valer sus reglas; pero deja de serlo cuando surge otra potencia con recursos de poder semejantes. En esa instancia, puede darse una transición de poder desde el Estado, hasta ahora dominante, hacia otra gran potencia. Esta transición puede resultar en guerra o puede darse de forma pacífica. Véase Jacek Kugler y A. F. K. Organski, "The Power Transition: A Retrospective and Prospective Evaluation", en Manus I. Midlarski (ed.), Handbook of War Studies, Boston, Unwin Hyman, 2006, pp. 171-194. 
El presente artículo se adentra, en primer lugar, en una descripción del actual orden internacional, así como en una conceptualización de poder medio emergente, como categoría derivada de la noción de potencia media. Posteriormente, se focaliza en tres ámbitos de gobernanza mundial: ${ }^{9}$ esfera comercial (Organización Mundial del Comercio, omc), esfera financiera (Banco Mundial, Fondo Monetario Internacional, G-20) y esfera medioambiental (Cumbres de Cambio Climático). De este modo, se busca establecer si efectivamente el papel de los poderes emergentes ha provocado modificaciones en los esquemas decisorios internacionales y cómo se han reconfigurado las relaciones entre las potencias tradicionales, las emergentes y las re-emergentes.

\section{LA ACTUAL ESTRUCTURA DE PODER INTERNACIONAL: ORDEN MUNDIAL Y PODERES EMERGENTES}

En el marco de la post-Guerra Fría, el mundo académico ha discutido extensamente sobre las transformaciones que están ocurriendo en el sistema internacional y sus posibles efectos en la distribución del poder global. Pese al paso de los años, y luego de casi 25 años del fin de la Guerra Fría, la mayoría de los estudiosos sigue considerando el actual sistema internacional como un orden "en transición". Esta amplia definición pone de manifiesto la existencia de múltiples conceptualizaciones: momento unipolar, ${ }^{10}$ sistema unipolar, ${ }^{11}$

${ }^{9}$ La gobernanza mundial está asociada a la ausencia de un gobierno formal de alcance global, pero también a la presencia de una estructura que es operativa gracias a la existencia efectiva de reglas, normas e instituciones permanentes que han emergido en muchas áreas de la vida internacional del nuevo siglo. Cf. José Antonio Sanahuja, "El G-20 y la Gobernanza Económica Global: cuestiones de Representatividad, Legitimidad y Eficacia”, Anuario Sociolaboral, núm. 2, Fundación 1 de mayo, 2012, pp. 197-213.

${ }^{10}$ Charles Krauthamer, "The Unipolar Moment Revisited", The National Interest, invierno de 2002-2003, pp. 5-17.

${ }^{11}$ John Ikenberry, John, Michael Mastanduno y William C. Wohlforth, "Introduction. Unipolarity, State Behavior and Systemic Consequences", World Politics, vol. 61, núm. 1, 2009, p. 3. 
unimultipolar, ${ }^{12}$ no polar, ${ }^{13}$ interpolar, ${ }^{14}$ retrowestfaliano, ${ }^{15}$ entre otros.

Lo cierto es que la vieja idea de una pirámide mundial -propia de la Guerra Fría- comenzó a desdibujarse. Desde esta perspectiva, el mundo ya no puede entenderse sobre la base de un modelo de "hub and spokes" ('eje y rayos'), en el cual todas las unidades remiten a un centro, puesto que la nueva configuración de fuerzas se acerca cada vez más a la imagen de un "mapa de metro", con innumerables líneas interconectadas y múltiples estaciones, si bien algunas son más importantes que otras. ${ }^{16}$

Aunque Estados Unidos sigue siendo la única superpotencia mundial (especialmente en términos estratégico-militares) su posición hegemónica se ha erosionado. Este proceso se explica por el protagonismo de ciertos estados que comenzaron a participar crecientemente en los procesos decisorios mundiales, junto a nuevos actores -originados tanto dentro, como fuera de las fronteras estatales- que se incorporaron activamente en el plano global.

En cuanto al concepto de poder emergente, como ya se mencionó, es una categoría que deriva de la noción de potencia media. Cabe recordar que este último tuvo un primigenio y significativo desarrollo en Canadá y en Australia, puesto que desde finales de la Segunda Guerra Mundial, y en vistas a la conformación de Naciones Unidas, los gobiernos de ambos países llevaron adelante una férrea defensa del papel de sus respectivos estados como potencias medias. En esas naciones entonces (o en muchas ocasiones

12 Samuel Huntington, "The Lonely Superpower", Foreign Affairs, vol. 78, núm. 2, 1999, pp. 35-49.

13 Richard Haas, "La era de la no polaridad. Lo que seguirá al dominio de Estados Unidos”, Foreign affairs: Lationamérica, vol. 87, núm 3, 2008, pp. 66-78.

14 Giovanni Grevi, "El mundo interpolar", Foreign Policy en español, abril-mayo de 2010, en http:/ / www.fp-es.org/el-mundo-interpolar

15 Alberto Rocha y Daniel Morales, "El sistema político internacional de posGuerra Fría y el rol de las potencias regionales mediadoras”, Espiral. Estudios de Estado y Sociedad, vol. 15, núm. 43, 2008, pp. 23-75.

16 Celso Amorim, "Brazilian Foreign Policy under president Lula (20032010): an overview", Revista Brasileira de Política Internacional, vol. 53, edición especial, 2010, pp. 214-240. 
relacionadas con ellas) se realizaron importantes contribuciones teóricas sobre el tema. ${ }^{17}$

Durante los primeros años de Guerra Fría, los poderes medios pertenecían a la esfera de influencia norteamericana. Eran occidentales, por lo general nórdicos, desarrollados y defensores de los sistemas de Naciones Unidas y de Bretton Woods. ${ }^{18}$ En este sentido, las llamadas potencias medias tradicionales, desarrolladas o de primera generación ${ }^{19}$ incluían países como Canadá, Australia, Noruega, Suecia, Austria, Suiza y Nueva Zelanda.

Las potencias medias desarrolladas cumplieron fundamentalmente, durante esta etapa, dos funciones: a) articulando nuevas ideas y liderando las negociaciones en aquellos temas sociales o económicos en los que las superpotencias no demostraban un interés especial y directo; $b$ ) ejerciendo una influencia moderadora de las tensiones entre las superpotencias en el contexto Este-Oeste

${ }^{17}$ Véanse Robert Mackay, "The Canadian Doctrine of the Middle Powers", en Harvey Dyck y Peter Krosby (eds.), Empire and nations. Essays in honour of Frederic Soward, Toronto, University of Toronto Press, 1969; Carsten Holbraad, Las potencias medias en la política internacional, México, FCE, 1989; Andrew Cooper, Richard Higgott y Kim Nossal, Relocating Middle Powers: Australia and Canada in a Changing World Order, Vancouver, ubc Press, 1993; María Cristina Rosas, "El estudio de las potencias medias en las Relaciones Internacionales: los casos de Australia y Canadá”, Relaciones Internacionales, núm. 78, 1998; Adam Chapnick, "The middle power”, Canadian Foreign Policy, vol. 7, núm. 2, 1999, pp. 73-82; Andrew Cooper, "The evolution of multilateralism in an intermediate state: the re-orientation of Canadian strategy in the economic and security arenas", en Paths to Power; Foreign Policy Strategies of Brazil, Canada, India and Mexico, Working Paper, Woodrow Wilson International Center, 13 de mayo de 1999; Andrew Hurrell, "Some Reflections on the Role of Intermediate Powers in International Institutions", Working Paper 244, "Paths to Power: Foreign Policy Strategies of Intermediate States", Washington, DC, Latin American Program, Woodrow Wilson International Centre, 2000, en http:/ / www.corteidh.or.cr/tablas/15011.PDF; Ronald Behringer, "Middle power leadership on human security", paper presented at the annual meeting of the Canadian Political Science Association, Nova Scotia, 2003, en http://www.cpsa-acsp. ca/paper-2003/behringer.pdf; David Dewitt, "Los poderes medios y la seguridad regional”, en Juan Tokatlian (comp.), India, Brasily Sudáfrica: el impacto de las nuevas potencias regionales, Buenos Aires, Libros del Zorzal, 2007, pp. 55-96.

${ }^{18}$ David Dewitt, art. cit., p. 57.

19 Ibidem., p. 9. 
("bridge-builders") con el objeto de lograr consensos y conservar la paz bajo los auspicios de Naciones Unidas. ${ }^{20}$

Posteriormente, durante el periodo de distensión, y ante la relajación de tensiones entre ambas cabezas de bloque, se produjo la aparición de nuevas potencias medias, las cuales "encabezaban la ofensiva del Tercer Mundo, demandaban un nuevo orden económico internacional, promovían la no-alineación y el fin de los bloques, levantaban temas como la paz y la igualdad en las relaciones internacionales". ${ }^{21}$ Este segundo grupo de potencias en desarrollo o de segunda generación incluyó a India, México, Nigeria, Brasil, Argentina, Indonesia y Yugoslavia e implicó un renovado interés académico por el tema. ${ }^{22}$ Teniendo en cuenta que además poseían cierto peso demográfico, económico y militar en sus respectivas áreas de pertenencia geográfica, consolidaron espacios de influencia regional, por lo cual también se las llamó entonces potencias regionales. ${ }^{23}$

A partir de la post-Guerra Fría, especialmente luego de los atentados a las Torres Gemelas en 2001, el papel de las potencias medias desarrolladas comenzó a erosionarse. Gobiernos como los de Canadá o Australia continuaron basándose en un discurso en el cual se autorreconocían como poderes medios, pero esta

${ }^{20}$ Carsten Holbraad, op. cit.; Ronald Behringer, documento citado; David Dewitt, art. cit.

${ }^{21}$ José Miguel Insulza, "El dilema de una potencia intermedia", Nexos, núm. 102, 1 de junio de 1986, en http:/ /www.nexos.com.mx/?p=4630

22 Véanse Leopoldo González Aguayo, "Aproximación a una teoría de las potencias medianas”, Relaciones Internacionales, vol. 3, núm. 8, 1975; Carlos Pérez Llana, “¿Potencias intermedias o países mayores? La política exterior de la Argentina, el Brasil y México”, en Luciano Tomassini (comp.), Relaciones Internacionales de la América Latina, México, FCE, 1981; José Miguel Insulza, art. cit.; Guadalupe González, "Incertidumbres de una potencia media regional: las nuevas dimensiones de la política exterior mexicana”, en Olga Pellicer (comp.), La política exterior en México: desafíos en los ochenta, México, cIDE, colección Estudios Políticos, enero de 1983; Jordi Palou, "El concepto de potencia media. Los casos de España y México", Revista CIDOB d'Afers Internacionals, núm. 26, 1993, pp. 7-35; Paulo Fagundes Visentini, "O Brasil como Potência Média: Possibilidades de ação internacional", en Ricardo Sennes (org.), O Brasil e a Política Internacional, São Paulo, IDEsP, 1998.

${ }^{23}$ Esther Barbé, op. cit., p. 150; Guadalupe González, art. cit. 
retórica quedó crecientemente desconectada de sus efectivas acciones. ${ }^{24}$ Esta realidad ha llevado a catalogarlas como potencias estancadas. ${ }^{25}$

Como contrapartida, algunas de las potencias medias en desarrollo surgidas en los años setenta comenzaron a ser identificadas como economías o poderes emergentes. Sobre este punto, cabe diferenciar entre mercado emergente, ${ }^{26}$ economía emergente ${ }^{27}$ y poder emergente. Mientras los dos primeros se asocian a factores meramente económicos, especialmente a las tasas de crecimiento nacionales; el segundo concepto implica un enfoque más integral, incluyendo no sólo aspectos económicos, sino también elementos vinculados a la política doméstica y a la proyección internacional de un determinado país.

${ }^{24}$ En este sentido resulta apropiado mencionar algunos ejemplos. Con respecto a la función de las potencias medias tradicionales como articuladoras de iniciativas en distintos ámbitos multilaterales, cabe mencionar la pérdida de protagonismo de Australia en la oмc. En efecto, a partir de 2003 se produce la conformación del G-20 a instancias de Brasil, en perjuicio del Grupo CAIRns, que se había creado por iniciativa de Australia. En cuanto al papel de las potencias medias tradicionales como mediadoras en conflictos, Dewitt, art. cit., asegura que "la división ideológica del mundo entre capitalismo y comunismo ha sido reemplazada por una nueva división entre el fundamentalismo islámico y el secularismo liberal”. El autor considera que las potencias medias tradicionales no se encuentran capacitadas para actuar como puentes entre estas dos visiones opuestas del sistema internacional.

${ }^{25}$ Detlef Nolte, "Potencias regionales en la política internacional: conceptos y enfoques de análisis", German Institute of Global and Area Studies (GIGA), núm. 30, octubre de 2006, en http://www.giga-hamburg.de/content/publikationen/pdf/wp30_nolte.pdf

${ }^{26}$ En 1997, Jeffrey Garten (The Big Ten: The Big Emerging Markets and How They Will Change Our Lives, Nueva York, Basic Books, 1997, p. 18) identificó diez grandes mercados considerados emergentes a partir de la década de 1990. En Asia: China (incluyendo a Hong Kong y Taiwán), Corea del Sur, Indonesia e India; en África: Sudáfrica; en Europa: Polonia y Turquía; y en América Latina: México, Brasil y Argentina.

27 En 2001, Jim O’Neill (documento citado), economista perteneciente al grupo inversor Goldman Sachs, dio a conocer la llamada "tesis BRIC", en que identifica cuatro economías emergentes -Brasil, Rusia, India y China- en función de sus posibilidades económicas. 
En este contexto, el tratamiento académico de la cuestión aquí planteada se reposicionó a principios del siglo Xxi. Diversos autores comenzaron a destacar el papel de estos nuevos poderes, desde apelaciones más generales como "el ascenso del resto" 28 o "el Segundo Mundo" 29 hasta teorizaciones más puntuales sobre el desempeño de los poderes emergentes en una diversa gama de cuestiones: seguridad, ${ }^{30}$ comercio, ${ }^{31}$ asuntos sociales ${ }^{32}$ o vinculados al cambio climático global. ${ }^{33}$

Desde un punto de vista rigurosamente conceptual, se han empleado diversas categorías. "Países intermedios", 34 "potencias

28 Como Alice Amsden, A ascensao do “resto", São Paulo, Ed. UnesP, 2001, y Fareed Zakaria, The post-American world, Nueva York, Norton, 2009.

${ }^{29}$ Parag Khana, El segundo mundo: Imperios e influencias en el nuevo orden global, Nueva York, Random House, 2008.

${ }^{30}$ Alcides Costa Vaz (ed.), Intermediate status, regional leadership and security: India, Brazil and South Africa, Brasilia, Editora Universidade de Brasilia, 2006.

${ }^{31}$ Dot Keet, Alternativas estratégicas Sur-Sur al sistema económico y régimen de poder globales, Ámsterdam, Trasnational Institute (TNI), 2006.

32 Khatchik Derghougassian, "IBSA no gubernamental: movilización social, diplomacia ciudadana y gobernabilidad de seguridad en la integración Sur-Sur", en Juan Tokatlian (comp.), op. cit., pp. 191-238; Clarisa Giaccaglia, "Condicionantes sociales en el proceso de formación de potencias mundiales. Un análisis de los países de IBSA a partir de un recorrido histórico", Relaciones Internacionales, núm. 15, octubre de 2010, pp. 33-60, y "El rol de India, Brasil y Sudáfrica en el sistema internacional contemporáneo: ¿qué tipo de desarrollo y para quién?”, Revista Ciência Ė Trópico, vol. 35, núm. 2, 2011, pp. 367-415.

${ }^{33}$ Eduardo Viola, "Climate governance in an international system under conservative hegemony: the role of major powers" Revista Brasileira de Politica Internacional, vol. 55, núm. especial, 2012, pp. 9-29; Andrew Hurrell y Sandeep Sengupta, "Emerging powers, North-South relations and global climate politics", International Affairs, vol. 88, núm. 3, 2012, pp. 463-484.

34 Andrew Hurrell, documento citado; Ricardo Sennes, "Países intermediários e fóruns multilaterais: algumas considerações”, trabajo presentado en el $3^{\circ}$ Encontro Nacional da AвсP, Associação Brasileira de Ciência Política, Niteroi, 28 a 31 de julio de 2002; María Regina Soares De Lima y Mónica Hirst, "Brasil como país intermediário e poder regional”, en A. Narlikar, A. Hurrell, M. R. Soares De Lima, M. Hirst, N. Macfarlane y R. Foot (comps.), Os BRICS e a Ordem Global, Río de Janeiro, FGV Editora, 2009. 
regionales" ${ }^{35}$ y "poderes emergentes", ${ }^{36}$ han sido las más utilizadas para hacer referencia a estos nuevos poderes en ascenso. Sin embargo, la elaboración de definiciones teóricas ha sido comparativamente escasa. En este sentido, es posible destacar las aportaciones de Detlef Nolte, quien propone un concepto analítico de potencia regional. El autor destaca los atributos de poder y las posibilidades de actuación de esta clase de estados en un ámbito geográfico determinado. De este modo, enfatiza la presencia de liderazgos regionales y el desempeño de estas potencias como representantes de los intereses de sus respectivas áreas de pertenencia. ${ }^{37}$ Sin embargo, resulta importante destacar que no todas las potencias medias regionales adquirieron mayor protagonismo en los inicios del nuevo siglo. En efecto, sólo un subgrupo de "grandes países en desarrollo"38 irrumpió con un alto perfil internacional en el escenario mundial. De allí el carácter de su "emergencia" en múltiples esferas globales de negociación. En sentido estricto, se trata entonces de poderes medios emergentes.

En cuanto al desempeño en el marco del binomio "esferas regionales / globales", 39 el accionar de estos nuevos poderes presenta la particularidad de una doble actuación, es decir es indudable que la posesión de una esfera de influencia regional es una condición

35 Detlef Nolte, "How to compare regional powers: analytical concepts and research topics”, Review of International Studies, vol. 36, núm. 4, 2010, pp. 881-901; David Cooper, "Somewhere Between Great and Small: Disentangling the Conceptual Jumble of Middle, Regional, and «Niche» Powers", The Journal of Diplomacy and International Relations, vol. 14, 2013, pp. 23-35, entre otros.

36 Varun Sahni, “¿Ancla flotante o plataforma de lanzamiento? Dinámica regional de los poderes emergentes” en Juan Tokatlian (comp.) op. cit., pp. 97-126; Andrew Cooper y Agata Antkeiwicz (eds.), Emerging Powers and Global Governance, Waterloo, Wilfried Laurier University Press, 2008; Andrew Hurrell, "Hegemonía, liberalismo e ordem global: qual é o espaço para potencias emergentes?”, en A. Narlikar, et al. (comps.), op. cit., pp. 9-41; Susanna Vogt (coord.), Growth and Responsibility. The Positioning of emerging powers in the global governance system, Sankt Augustin-Berlín, Konrad-Adenauer-Stiftung, 2009, entre otros.

37 "Potencias regionales en la política internacional...".

${ }^{38}$ Se trata de estados con importante peso territorial, demográfico, económico y, en algunos casos, militar.

${ }^{39}$ Para un mayor análisis sobre este tema, se recomienda ver D. Nolte, "How to compare regional powers...” y D. Cooper, art. cit. 
necesaria para la consideración de un estado como poder medio emergente. Pero también es cierto que un poder medio emergente puede en diversas ocasiones desenvolverse en ámbitos globales de manera independiente a su respectiva zona de proyección regional, trascendiendo los límites de una mera representación geográfica. En este sentido, se coincide con lo sostenido por Andrew Hurrell, quien asegura que "hay una opinión común de que los actores globales emergentes también serán potencias regionales y que su estatus como líderes regionales es un elemento importante de su estatus global". ${ }^{40}$ El autor advierte que "esta opinión es incorrecta desde el punto de vista histórico. Algunas de las grandes potencias más exitosas, Reino Unido y Estados Unidos, tuvieron éxito, precisamente porque lograron evitar quedar atrapadas dentro de sus respectivas regiones y pudieron establecer los términos para sus compromisos continentales". ${ }^{41}$

En síntesis, un poder medio emergente es una potencia media que necesariamente posee un ámbito de influencia regional ${ }^{42} \mathrm{y}$, al mismo tiempo, un emergente con gran exposición global que no necesariamente representa, en todos los casos, los intereses de su región. ${ }^{43}$ Esto es lo que David Cooper define como un overlapping concept. ${ }^{44}$ Por tanto, la conceptualización propuesta en este trabajo, sostiene que un poder medio emergente puede definirse como aquel Estado que sobre la base de significativas capacidades materiales desarrolla un creciente activismo internacional en ámbitos multilaterales, tanto en su entorno regional más próximo, como en sucesivas y simultáneas negociaciones globales. Asimismo, se autopercibe y es percibido por otros agentes como diferente tanto de las restantes unidades estatales (pequeñas o iguales), como de las grandes potencias tradicionales.

40 "Brasil y la tormenta que se avecina”, Foreign Affairs: Latinoamérica, vol. 9, núm. 2, 2009, p. 48.

${ }^{41}$ Loc. cit.

42 Canadá sería un ejemplo de potencia media, sin ámbito de influencia regional.

${ }^{43}$ Etiopía sería un ejemplo de potencia regional en África Oriental, pero con una presencia en foros globales de negociación prácticamente inexistente.

44 Art. cit., p. 29. 
La definición detallada líneas arriba engloba los principales postulados esgrimidos por las corrientes teóricas más importantes de la disciplina de las Relaciones Internacionales. Incluye tanto factores materiales (principal preocupación del realismo), aspectos procedimentales y de dinámica relacional entre agentes (foco de interés de los liberales institucionalistas), como el tema de las expectativas recíprocas (cuestión especialmente considerada por los constructivistas). ${ }^{45}$ Este concepto puede entonces ser analíticamente desagregado en tres dimensiones: capacidades materiales, activismo internacional y percepciones (propias y ajenas). Ahora bien, ¿quiénes son, hoy en día, estas potencias medias del sistema mundial y quiénes, en particular, están emergiendo?

\section{Potencias MEdias y PODERES MEDIOS EMERGENTES \\ EN EL SIGLO XXI}

Sobre la base de las conceptualizaciones efectuadas en el apartado anterior, es posible mencionar, en primer lugar, un listado estimativo de las actuales potencias medias del orden internacional, entre las cuales se encuentran: México, Corea del Sur, España, Canadá, Indonesia, Turquía, Irán, Australia, Taiwán, Polonia, Argentina, Países Bajos, Arabia Saudita, Tailandia, Egipto, Pakistán, Colombia, Malasia y Nigeria. ${ }^{46}$ Asimismo, y a partir de la actuación del G-20 financiero frente a la crisis económica de 2008, es posible destacar, en este primer grupo, la actuación de México, Argentina, Arabia Saudita, Turquía, Corea Del Sur, Australia e Indonesia.

En cuanto a los poderes medios emergentes, se incluye a la mayoría de los países miembros de BRICs, esto es Brasil, India, China y Sudáfrica. El caso de Rusia merece una explicación especial.

45 Un análisis pormenorizado de la conceptualización de los poderes emergentes es el de la tesis doctoral de C. Giaccaglia, Estrategias de política exterior de los poderes medios emergentes en el escenario internacional de la post-Guerra Fría. El caso de IBSA-India, Brasil y Sudáfrica (2003-2010), Rosario, Universidad Nacional de Rosario, Argentina, 2012.

46 Central Intelligence Agency, The World Factbook, en https://www.cia.gov/ library/publications/the-world-factbook/ 
Dado que este país euroasiático fue el eje sobre el cual se forjó la Unión Soviética, una de las superpotencias durante la Guerra Fría, resulta importante diferenciarla del resto de los poderes medios emergentes, puesto que su verdadera situación se asocia en mayor medida a un proceso político que busca su "reemergencia" en el orden internacional.

Con respecto a Sudáfrica bien vale señalar que, históricamente, se ha desempeñado como una importante potencia regional del África subsahariana. ${ }^{47}$ Sin embargo, su incorporación a IBSA, en primer lugar, y luego a BRICS, la catapultó a los principales ámbitos globales de discusión multilateral. Por tanto, su emergencia responde, en menor medida, a sus atributos materiales de poder, que son incomparables a los de China, India o Brasil. Empero, su mayor fortaleza reside en el hecho de ser considerada por las restantes grandes potencias del escenario mundial como la principal y, muchas veces, única representante del continente africano en las esferas globales de negociación. En otros términos, el aspecto relativo a las percepciones propias y, sobre todo, ajenas -en el marco de la definición de poder medio emergente ya señalada-constituye en este caso un elemento decisivo para la inclusión de Sudáfrica en este grupo de poderes en ascenso.

En relación con las variables sistémicas que facilitaron la irrupción de estos nuevos poderes, cabe señalar que Estados Unidos, en virtud de su condición de superpotencia mundial, desarrolló una política exterior post-11-S, caracterizada por conductas unilaterales. Este accionar implicó además desentenderse de sus compromisos globales, con excepción de las áreas que consideraba estratégicas para su interés nacional. La situación descrita ofreció un marco de oportunidades para que algunas potencias medias del mundo en desarrollo incrementaran sus niveles de actuación y responsabilidad en las esferas globales.

47 Gladys Lechini, "Sudáfrica: la identidad nacional como construcción y el liderazgo regional como destino manifiesto" en Anabella Busso (comp.), Fuerzas profundas e identidad. Reflexiones en torno a su impacto sobre la política exterior. Un recorrido de Casos, Rosario, UnR editora, 2010, pp. 175-188. 
En efecto, la llegada de nuevas administraciones, desde el año 2003 en adelante, a los gobiernos de Brasil, India y Sudáfrica puso de manifiesto la voluntad política de desarrollar una presencia internacional más activa, tal como quedó demostrado con la asunción de Lula da Silva en Brasil, Manmohan Singh en India y Thabo Mbeki (luego Jacob Zuma) en Sudáfrica. La pretensión de un bien delimitado perfil internacional se convirtió en una intensa participación de estos poderes emergentes en diversas esferas globales de negociación. En el caso de China, en cambio, desde el gobierno de $\mathrm{Hu}$ Jintao se cultivó un ascenso con bajo perfil para intentar convencer de que la emergencia político-económica del gigante asiático no constituye una amenaza para el resto del sistema mundial.

A la luz de los interrogantes aquí planteados, este artículo se concentra en el análisis de la segunda dimensión mencionada en la definición de poder medio emergente propuesta, esto es activismo internacional, puesto que por el comportamiento político-diplomático ejercido por estos poderes en los ámbitos globales será posible aproximarnos a la lógica actual de poder internacional instaurada entre poderes tradicionales y emergentes.

EL PAPEL NEGOCIADOR DE LOS PODERES EMERGENTES: POLÍTICAS EXTERIORES PROACTIVAS Y ALTO PERFIL EN LAS ESFERAS DE DECISIÓN MUNDIAL

Desde la perspectiva del liberalismo institucional, los ámbitos multilaterales se han convertido en una esfera vital para el desempeño internacional de los poderes medios emergentes. Las esferas de negociación, regionales y globales, representan la oportunidad de contar con un espacio político, en el cual pueden orientar las normas emergentes según propios intereses, esto es desestimando aquellas reglas o procedimientos que pueden afectarlos o promoviendo aquellas que pueden favorecerlos. Esta práctica ha sido notoriamente desarrollada por los poderes medios emergentes con desempeños altamente satisfactorios en múltiples ámbitos de discusión global, lo cual permitió a países como Brasil, India, China y Sudáfrica participar en prácticamente todas las negociaciones 
internacionales de relevancia ocurridas durante las últimas décadas. Para este trabajo, cabe destacar dos coaliciones surgidas en la primera década del nuevo siglo: IBSA y BRICs. ${ }^{48}$

Es de recordar que en el año 2001 el economista Jim O'Neill, perteneciente a la consultora internacional Goldman Sachs, acuñó el acrónimo BRICs, haciendo referencia a Brasil, Rusia, India y China y a las potencialidades económicas que estas cuatro economías presentaban. ${ }^{49}$ Este concepto adquirió gran notoriedad tanto en los medios periodísticos, como en las audiencias en general, sobre la base de su aparente "novedad" en cuanto a la aparición de nuevos poderes que vendrían a reemplazar a los ya existentes. Sin embargo, la iniciativa no tuvo, en un principio, respaldo gubernamental, puesto que su principal objetivo radicaba en orientar a los inversionistas trasnacionales hacia buenos negocios.

Como un proceso paralelo, el 6 de junio de 2003 se produjo por iniciativa de Brasil la creación de IBSA, coalición gubernamental conformada con el propósito de potenciar la presencia de India, Brasil y Sudáfrica en el escenario internacional. A lo largo de los años, el grupo coordinó posiciones en múltiples esferas de negociación global como Naciones Unidas, la OMC, la OMPI, las Cumbres de Cambio Climático, el FMI, la FAO, la oms y la oIT, entre otras. De igual manera, el bloque dio a conocer, por medio de distintos documentos oficiales, su posición conjunta frente a los conflictos bélicos

${ }^{48}$ La conformación de estas iniciativas responde a un largo proceso de activismo diplomático desarrollado por los países del hoy en día denominado Sur Global. Este recorrido abarca desde la actuación a partir de los años sesenta en el Movimiento de Países No Alineados y en el G-77, pasando luego en los años ochenta por la creación, por ejemplo, del G-10, en el marco del GATT, y del G-15, en el proceso de la deuda externa. Una variable constante en todo este recorrido refiere a la repetida presencia de Brasil, China, India y Sudáfrica como promotores o líderes de estas coaliciones. Para más detalles, véase C. Giaccaglia, op. cit., pp. 164-171.

${ }^{49}$ Las proyecciones realizadas por este grupo inversor indicaron que para el año 2050, la economía de China pasaría a ocupar el primer lugar, dejando detrás a Estados Unidos. Además, India, Brasil y Rusia quedarían también ubicados dentro de los primeros seis puestos a nivel mundial. Véase J. O’Neill, “Dreaming with BRICs: The Path to 2050", GS Global Economics, Paper 99, noviembre de 2003, en http:// www.goldmansachs.com/our-thinking/archive/archive-pdfs/brics-dream.pdf 
más importantes de los últimos años, tales como Medio Oriente, Irak, Afganistán, Libia y Siria.

Tiempo más tarde, en 2008, BRICs se transformó también en una iniciativa gubernamental que incluye a los gobiernos de Brasil, Rusia, India, China y, a partir de 2011, a Sudáfrica. Sin dudas, la crisis financiera internacional desencadenada ese mismo año incentivó esta decisión, teniendo en cuenta que el bloque exhortó por una mayor participación de sus estados miembros en las decisiones de gobernabilidad económica internacional. Esta demanda colectiva fue, además, uno de los factores que impulsó la inmediata reconfiguración del G-20 financiero. ${ }^{50}$

Durante sus primeros años de funcionamiento, BRICs se concentró especialmente en cuestiones de índole financiera tendientes a promover una reforma de la vigente arquitectura global. En los últimos años, empero, y considerando una serie de conflictos bélicos que acentuaron su importancia en el escenario mundial, el grupo se ha preocupado también por consolidar una posición común ante los que enfrentan Medio Oriente, Libia o Siria. En suma, los poderes medios emergentes se han distinguido crecientemente en los últimos años, convirtiéndose en interlocutores válidos y cada vez más necesarios para el establecimiento del orden internacional.

La actual estructura de poder mundial manifiesta dos elementos que han contribuido a estas transformaciones. Por un lado, la interconexión global derivó en mayores grados de institucionalización mundial, lo cual resultó en una regulación multilateral internacional cada vez más invasiva de espacios que tradicionalmente eran exclusivos de las esferas estatales. Esta situación exigió un duro aprendizaje técnico-diplomático para los países en desarrollo, para evitar verse perjudicados por las negociaciones realizadas sobre múltiples asuntos en variadas esferas globales. En este sentido, poderes medios emergentes como China, India, Brasil y Sudáfrica y re-emergentes como Rusia demostraron estar a la altura de las circunstancias para poder sentarse en

50 Cabe recordar que, a partir de 2008, el G-20 financiero comienza a reunir el más alto nivel político, en que participan los jefes de Estado y de gobierno de todos sus países miembros. 
una misma mesa de negociación junto con el club de los grandes poderes tradicionales (otrora reunidos en el G-7). Por otro, el orden mundial presenta paradójicamente mayores grados de informalidad. En efecto, las instituciones internacionales multilaterales, centros históricos del debate global (Naciones Unidas, Banco Mundial, FMI, OMC) perdieron legitimidad en los últimos años y comenzaron a convivir con otras instancias de diálogo informal, en las cuales los poderes medios emergentes encontraron mejores vías de acceso ( $\mathrm{G} 7+\mathrm{O} 5^{51}$ en un primer momento, luego G-20 financiero y más tarde IBSA/BRICs). En consecuencia, los poderes medios emergentes han actuado indistintamente, por dentro o por fuera de las organizaciones internacionales, según las circunstancias de negociación.

Asimismo, el ámbito formal de discusión que corresponde a cada temática de la agenda internacional se ha diluido significativamente. Esta situación da cuenta de un gran proceso negociador que atraviesa todas las instancias formales e informales y donde los temas son de naturaleza interméstica, se yuxtaponen y se vinculan entre sí en los distintos ambientes de discusión global. El rediseño del G-20 financiero aparece como el ejemplo paradigmático. La crisis financiera de 2008 benefició a las potencias emergentes permitiéndoles incorporarse en el seno de un grupo que se postuló como el punto de partida para una reconfiguración del orden mundial. En cuanto que miembros del G-20, los poderes emergentes pudieron demostrar su peso político-económico, así como el hecho de que representan diferentes regiones del mundo.

Cabe también preguntarse cuál es la estrategia de las grandes potencias tradicionales ante la emergencia de estos nuevos poderes. Teniendo en cuenta a Estados Unidos, Fareed Zakaria recomienda sobre la necesidad de que el gobierno norteamericano facilite la integración de los poderes emergentes en los principales órganos decisorios políticos y económicos internacionales. Esta nueva función, asegura, es muy diferente del papel tradicional de

${ }^{51}$ Diálogo informal que se estableció durante la presidencia del Reino Unido en 2005, entre los miembros del G-7 y el llamado Outreach 5 (O-5) conformado por Brasil, China, India, México y Sudáfrica. 
superpotencia e implica consulta, cooperación y compromiso. El poder deriva, en este sentido, de establecer los temas de agenda, definir las cuestiones y movilizar coaliciones: "It is not a top-down hierarchy in which the United States makes its decisions and then informs a grateful (or silent) world. But it is a crucial role because, in a world with many players, setting the agenda and organizing coalitions become primary forms of power". ${ }^{52}$ Zakaria agrega: "in an age of rising new powers, the United States' overriding goal should be the latter, so that even as these countries get more powerful, they will continue to live within the framework of the current international system". ${ }^{53}$ De este modo, Estados Unidos podrá evitar que los poderes emergentes actúen de forma independiente, esto es según sus propios intereses, pudiendo desestabilizar el sistema en su conjunto.

En este mismo sentido Daniel Drezner sostiene que, en el actual sistema internacional, Estados Unidos debe "mantener cerca a los viejos amigos del país, y a sus nuevos amigos, aún más cerca”. Caso contrario, las potencias emergentes podrían crear organizaciones internacionales alternativas que podrían ir en contra de los intereses norteamericanos. ${ }^{54}$ Por tanto, la estrategia de las grandes potencias tradicionales, especialmente de Estados Unidos pero también de los miembros del G-7, ha sido una política de creciente incorporación de los poderes medios emergentes en los ámbitos de decisión multilaterales con el propósito de poder influir e incluso condicionar sus comportamientos para que sus eventuales acciones sean más predecibles y calculables.

Para poder establecer si efectivamente el papel de los emergentes ha provocado modificaciones en los esquemas decisorios internacionales, los posteriores apartados se concentran en tres ámbitos de gobernanza mundial: esfera comercial, financiera y medioambiental.

52 Op. cit., p. 233.

53 Ibid., pp. 238-239.

${ }^{54}$ Daniel Drezner, "El nuevo orden mundial nuevo", Foreign Affairs: Latinoamérica, vol. 7, núm. 3, pp. 153-163. 
Esfera comercial: la OMC

El ámbito de la omc fue uno de los primeros espacios en los que los poderes medios emergentes desarrollaron una presencia preeminente. La conferencia ministerial de Cancún en 2003 constituyó el punto de partida con una destacada actuación de los países miembros de IBSA, especialmente de Brasil, que promovió la creación del G-20 agrícola. El abierto desafío de este grupo a las políticas agrarias de los países centrales alteró el clima general de las negociaciones, convirtiéndolo en un actor que Estados Unidos y la Unión Europea tuvieron que empezar a tomar en consideración. El surgimiento del G-20 demostró que la vieja estructura de poder y toma de decisiones en la omc era obsoleta y que era necesario expandir el círculo de poder a fin de reactivar la organización.

Lo cierto es que, aun con el paso de los años, las negociaciones no pudieron prosperar sino que, por el contrario, se ralentizaron significativamente. La búsqueda de un equilibrio aceptable, por parte de todos los estados miembros, entre agricultura y NAMA (productos no agrícolas) se transformó en la llamada "batalla grande". En efecto, todas las restantes negociaciones desarrolladas durante la ronda Doha se paralizaron a la espera del cierre de este gran tema. Asimismo, la persistencia de un contexto internacional convulsionado, producto del marco recesivo que prosiguió a la crisis financiera internacional en las regiones centrales, acentuó las divergencias entre los países miembros de este organismo.

El proceso negociador, además, frecuentemente se trasladó fuera de Ginebra con reuniones excluyentes que sólo contemplaron a un pequeño grupo, entre los cuales se destacan los FIPS (sigla con la que se conocen las llamadas "cinco partes interesadas": Estados Unidos, Unión Europea, Brasil, India y Australia). En esta circunstancia, cabe nuevamente diferenciar el papel de Rusia, quien a diferencia de los restantes poderes medios emergentes, ingresó a la omc en 2012 (diecinueve años después de haber solicitado su adhesión), por lo cual su situación dista en gran medida del resto de los miembros de BRICs.

Un nuevo capítulo comenzó para los emergentes a partir de 2013, cuando el brasileño Roberto Azevêdo fue elegido como 
Director general de la omc, convirtiéndose en el primer latinoamericano en acceder al cargo. En efecto, siete de los nueve candidatos que se postularon provenían de países en desarrollo o emergentes. ${ }^{55}$ Además, los países desarrollados no tuvieron un candidato propio en la instancia final de la selección. Por un lado, se podría afirmar que la elección de Carvalho de Azevêdo confirma la importancia creciente de los países emergentes en la esfera comercial multilateral. Por otro, el triunfo del candidato brasileño muestra un menor interés de los países desarrollados en buscar ejercer su influencia en la dirección de este organismo. "Por el contrario, éstos parecen estar más interesados en la negociación de los acuerdos mega-regionales como la Asociación Transpacífica (TPP) y, más recientemente, la Asociación Transatlántica de Comercio e Inversión (TTIP)" ${ }^{56}$

De este modo, la omc sigue siendo considerada un espacio de relevancia para la administración de los acuerdos alcanzados hasta la Ronda Uruguay y para la solución de diferencias al interior del régimen. Sin embargo, su importancia como foro de negociación entre los miembros ha decaído. Para los poderes tradicionales el riesgo de dejar la омc en manos de los emergentes "resulta muy reducido, en tanto que la omc sólo administrará lo ya acordado". ${ }^{57}$ En efecto, a partir de 2010, y a raíz de los serios estancamientos que presentaba la Ronda Doha, las cuestiones comerciales fueron incluidas en la agenda del G-20 financiero con el propósito de lograr el ansiado consenso entre las partes.

Con la llegada de Carvalho de Azevêdo, se intentó imprimir cierto dinamismo a la organización. Durante la novena conferencia

55 Alan John Kwadwo Kyerematen, de Ghana; Anabel González, de Costa Rica; Amina C. Mohamed, de Kenia; Ahmad Thougan Hindawi, de Jordania; Mari Elka Pangestu, de Indonesia; Tim Groser, de Nueva Zelandia; Taeho Bark de República de Corea, y Herminio Blanco, de México, junto a Roberto Carvalho de Azevêdo, de Brasil.

56 Uri Dadush, "La política comercial fortuita", Política Exterior, núm. 153, mayo-junio de 2013, en http://www.politicaexterior.com/articulos/politica-exterior/la-politica-comercial-fortuita/

57 Julieta Zelicovich, "Signos de cambio en el Régimen Multilateral de Comercio: análisis de la elección de Roberto Carvalho de Azevêdo como Director General de la oмc”, Boletim Mundorama, 2013. 
ministerial de la omc, realizada en Bali (Indonesia) en 2013, se alcanzó un acuerdo catalogado como "histórico", que tenía el propósito de simplificar los procedimientos burocráticos para impulsar el intercambio internacional de mercancías. Sin embargo, el acuerdo no logró prosperar por la oposición de India. El gobierno de este país adujo la falta de garantías que tiene el protocolo para salvaguardar la seguridad alimentaria de su población y solicitó una renegociación sobre este punto. ${ }^{58}$ Esta petición, sin embargo, fue rechazada por Estados Unidos y la Unión Europea. Los sucesos mencionados supusieron una nueva parálisis de la negociación y un duro revés para la ronda Doha lanzada hace ya dieciséis años y estancada desde 2008.

Pese a ello, los estados miembros del BRICs se siguen mostrando comprometidos con el logro de una economía global abierta. Reconocen, con todo, que los países tienen diferentes capacidades y se encuentran a diferentes niveles de desarrollo: "Reafirmamos nuestro apoyo a una apertura del comercio multilateral, inclusiva, no discriminatoria, transparente y un sistema basado en reglas al tiempo que seguiremos nuestros esfuerzos para la conclusión exitosa de la Ronda de Doha". ${ }^{59}$ Así, los poderes emergentes pretenden consolidar el espacio de la omc como el ámbito pertinente para la deliberación de las cuestiones comerciales internacionales, intentando circunscribir el ámbito de acción de los poderes centrales a este recinto. En definitiva, la situación demuestra un cuadro de tensión, en el cual las disímiles realidades sociales entre los poderes tradicionales y los emergentes impiden el ansiado acuerdo para un ordenamiento de la estructura de comercio global.

${ }^{58}$ India tiene un programa de subsidios por el cual compra productos a los pequeños agricultores por encima del precio del mercado, para después revenderlos, a precios ajustados, al poder adquisitivo de los ciudadanos más pobres. Este sistema ha sido duramente criticado por varios miembros de la oмc, especialmente por Estados Unidos, los cuales señalan que no es consistente con las normas de la organización.

59 BRICS, Declaración conjunta VI Cumbre, 2014, en http://www.mea.gov. in/bilateral-documents-es.htm?dtl/23635/Sixth+BRICS+Summit++Fortaleza+De claration 
Esfera FinANCiera (BAnco Mundial, FMi, G-20)

La crisis financiera internacional de 2008 puso de manifiesto la creciente incapacidad de las instituciones de Bretton Woods, para hacer frente a las nuevas realidades mundiales, ante lo cual, los BRICS exhortaron fuertemente por modificaciones en la estructura de la gobernabilidad económica internacional: "Acreditamos que o mundo precisa, hoje, de uma arquitetura financeira reformada e mais estável, que fará a economia global menos propensa a crises futuras e mais resistente a elas. O FMI e o Banco Mundial precisam urgentemente resolver seus déficits de legitimidade" ${ }^{60}$ Como consecuencia, el bloque propuso en 2010 modificaciones importantes para tales organismos multilaterales tendientes a: 1) una reforma del sistema de votos en favor de los poderes emergentes; 2) un método de selección, abierto y basado en el mérito, para los cargos directivos del FMI y del Banco Mundial; 3) la posibilidad de introducir una moneda de reserva alternativa al dólar o una nueva moneda global.

Años más tarde, en 2014 los gobiernos miembros de BRICS continuaron promoviendo cambios en la arquitectura financiera mundial a partir de la creación del Nuevo Banco de Desarrollo, ${ }^{61}$ -destinado a proyectos de infraestructura- y la puesta en marcha del Acuerdo de Reservas de Contingencia, ${ }^{62}$ orientado a ofrecer a

${ }^{60}$ BRICS, Declaración conjunta II Cumbre, 2010, pp. 2-3, en http://www.pravasitoday.com/wp-content/uploads/2011/04/BRICS-SUMMIT-2011-JOINT-DECLARATION1.pdf

${ }^{61}$ El Nuevo Banco de Desarrollo comenzará con un capital inicial de 50.000 millones de dólares, al que cada país aportará una quinta parte. Según fuentes diplomáticas se establecerá en Shanghai y en principio lo presidirá un brasileño. Financiará proyectos de infraestructura en los países miembros y luego dará préstamos también a otras naciones en desarrollo fuera del grupo. Cf. "Los BRICs quieren ser el motor del desarrollo", La Nación, Buenos Aires, 15 de julio de 2014, en http:/ / www.lanacion.com.ar/1710053-los-brics-quieren-ser-el-motor-del-desarrollo

${ }^{62}$ Este acuerdo de reservas estaría dotado en un principio por 100.000 millones de dólares, de los cuales China aportará 41.000 millones, Rusia, Brasil y la India 18.000 millones cada uno, y Sudáfrica, 5.000 millones. A diferencia del banco, que se financiará con contribuciones iguales de cada uno de sus miembros, el fondo refleja las diferencias en los niveles de reservas de los países miembros (loc. cit.). 
sus miembros, fondos de estabilización para hacer frente a dificultades en sus balanzas de pagos o a ataques especulativos contra sus monedas. Mientras que el primero se presenta con funciones similares a las del Banco Mundial, el segundo emula los principales objetivos del FMI. Los primeros resultados de todas estas proposiciones realizadas por los BRICs se dieron en torno al Banco Mundial. A principios de 2010, la organización crediticia multilateral introdujo una reforma en los derechos de voto, ${ }^{63}$ la cual otorgó mayor peso a los emergentes reconociendo su creciente importancia en la economía global.

Con respecto del FMI, también en 2010, se aprobó un programa de reformas de gran alcance del régimen de $\operatorname{cutatas}^{64} \mathrm{y}$ de su estructura de gobierno. Sin embargo, el congreso norteamericano se opuso durante varios años a realizar la ratificación de este acuerdo. Pese a estas demoras, la reforma del FMi fue finalmente aprobada por Estados Unidos a fines de 2015, por lo que el acuerdo ha entrado recientemente en vigor a partir del 26 de enero de 2016. Las modificaciones implementadas implican una importante reorganización de las cuotas para reflejar mejor la evolución del peso relativo de cada país miembro del organismo en la economía mundial, reforzando la representación de las potencias emergentes. De este modo, China se convierte en el tercer país miembro más grande del FMI mientras que Brasil, India y Rusia pasan a encontrarse entre los 10 principales accionistas de la organización. ${ }^{65}$

${ }^{63}$ La reforma de cuotas del Banco Mundial introdujo un aumento de 3,13 puntos porcentuales de los derechos de voto de los países en desarrollo y en transición (PDT), llevándolos al 47,19\%, lo que representa un traslado total de 4,59 puntos porcentuales de los derechos de voto a los PDT desde 2008. Cf. Banco Mundial, "El Banco Mundial introduce una reforma de los derechos de voto y obtiene un aumento por valor de US\$86.000 millones", comunicado de prensa, sitio web oficial, 25 de abril de 2010.

${ }^{64}$ La actual fórmula de cálculo de las cuotas en el FMI es un promedio ponderado del PIB (ponderación de 50\%), el grado de apertura (30\%), la variabilidad económica $(15 \%)$ y las reservas internacionales (5\%). La cuota determina en gran medida el número de votos que corresponde a un país miembro en las decisiones del FMI ("Las cuotas en el FMI", sitio web oficial, enero de 2016).

${ }^{65}$ Cabe destacar que la cuota de los EE.UU. en el FMI seguirá siendo mayoritaria, por lo que mantiene su capacidad de veto y sólo se verá reducida ligeramente, 
En cuanto a la estructura de gobierno, la reforma aprobada deja de lado la práctica consuetudinaria por la cual siempre se elegía un Director Gerente de nacionalidad europea, previendo que éste sea designado mediante la elección transparente de todos los miembros del Directorio Ejecutivo ${ }^{66}$ de la organización, el cual, luego de esta reforma, tiene dos representantes menos provenientes de países europeos. ${ }^{67}$

Tomando en consideración las posibles estrategias esgrimidas por las potencias tradicionales ante estos cambios -ya señaladas en el apartado anterior-, es posible pensar que tales poderes, especialmente Estados Unidos, decidieron finalmente aceptar y acelerar el proceso de reformas de las instituciones de Bretton Woods ante la inminente puesta en marcha del Nuevo Banco de Desarrollo de los BRICs, el cual comenzará a operar a partir de 2016. En efecto, con estas dos estructuras clave, el grupo podría encaminar la construcción de una arquitectura financiera global alternativa al Banco Mundial y al FMI y, por consiguiente, alternativa a la superpotencia norteamericana.

Estas percepciones de los poderes tradicionales podrían explicar también la decisión de rediseñar el funcionamiento del G-20 financiero a partir de 2008 con un reconocimiento implícito -a través de su convocatoria- del creciente papel de los países emergentes en la nueva distribución del poder económico y político global. En 2016, además, China asumirá la presidencia del G-20 y será entonces la primera vez que el gigante asiático encabece uno de los principales foros de cooperación económica global. En este marco de negociación, el gobierno chino pretende avanzar en el proceso de reformas con el objeto de obtener la inclusión del yuan en la cesta de divisas ${ }^{68}$ que el FMI utiliza como referencia para fijar los derechos especiales de giro. ${ }^{69}$

desde el $17,69 \%$ actual hasta el $17,40 \%$, seguida por Japón, que mantiene el segundo lugar (idem).

${ }^{66} \mathrm{El}$ director gerente del FMI es el presidente del Directorio Ejecutivo.

${ }^{67}$ FMI, "Cómo toma sus decisiones el FMI", ficha técnica, sitio web oficial, 27 de enero de 2016, en https://www.imf.org/external/np/exr/facts/spa/governs.htm

${ }^{68} \mathrm{La}$ actual cesta de divisas incluye el dólar, el euro, el yen y la libra.

${ }^{69}$ Alicia González, "Los brics y la gobernanza económica mundial”, Política 
El grado de coordinación y eficacia que puedan lograr las nuevas instituciones planteadas es lo que finalmente permitirá constatar cuán exitoso ha sido el camino propuesto por los BRICS y cuán dispuestos se encuentran a desempeñar un papel destacado en la gobernanza de la economía global.

\section{Esfera medioambiental: cumbres de cambio climático}

Las cumbres sobre cambio climático constituyen otro espacio en el cual los poderes emergentes tuvieron un papel destacado en los últimos años. Cabe recordar que en la Convención Marco de Naciones Unidas sobre Cambio Climático (CMNUCC) -acuerdo internacional celebrado en 1992 en Río de Janeiro- fue establecido el principio de "responsabilidades comunes pero diferenciadas", por el cual se dividió a los estados en dos grandes grupos con diferentes niveles de compromiso: "los países desarrollados"70 y "los países en desarrollo".

En virtud de este acuerdo, los países desarrollados eran los únicos obligados a adoptar políticas destinadas a reducir sus emisiones de gases de efecto invernadero (GEIs). Asimismo, los más industrializados de este primer grupo estaban obligados a ofrecer recursos financieros que permitiesen a los demás países emprender actividades de reducción de las emisiones contaminantes. Al segundo grupo de naciones en desarrollo, en cambio, sólo se les exigía compilar un inventario de las emisiones y presentar periódicamente informes sobre las medidas que estaban adoptando respecto al cambio climático. $^{71}$

Exterior, núm. 164, marzo-abril de 2015, en http:/ /www.politicaexterior.com/articulos/politica-exterior/los-brics-y-la-gobernanza-economica-mundial/

${ }^{70}$ Este primer grupo estaba formado por aquellos estados que eran miembros de la oCDE en 1992, más las denominadas "economías en transición" (naciones de la antigua órbita soviética).

${ }^{71}$ Convención Marco de Naciones Unidas sobre Cambio Climático, sitio web oficial: https:/unfccc.int/files/essential_background/background_publications_ htmlpdf/application/pdf/convsp.pdf 
$\mathrm{Al}$ paso de los años y las consiguientes transformaciones ocurridas en la distribución del poder político y económico mundial, comenzaron a discutirse nuevos esquemas. En ocasión de la llamada cop15, organizada en 2009, en Copenhague, se abrió el debate sobre si los grandes países en desarrollo, vueltos potencias emergentes, deberían también asumir compromisos de reducciones de gases. Esta discusión se hizo polémica, porque China se había convertido en el país que más contamina, superando a Estados Unidos. Sin embargo, cada chino emite la cuarta parte que un norteamericano. Por tanto, el proyecto de acuerdo de Copenhague buscaba crear una categoría intermedia de países que incluyera a los "grandes emergentes" e incitarlos a asumir compromisos de reducción.

En este contexto, se produjola formación del BASIC (IBSA+China). Este bloque se había comenzado a gestar al interior de IBsA, grupo que ratificaba su apoyo al principio de una responsabilidad común, pero diferenciada en relación con la responsabilidad histórica de los países industrializados. En las reuniones preparatorias, el BASIC acordó "cuáles serían las líneas rojas (o puntos no negociables) y también se decidió que se retirarían en conjunto de las negociaciones si los países desarrollados intentaban imponer sus condiciones". ${ }^{72}$ En suma, tras largas y en parte caóticas negociaciones, el Acuerdo de Copenhague no pudo ser aprobado por consenso. En los posteriores y sucesivos encuentros, los poderes emergentes siguieron manteniendo una posición común que se enfrentaba a las posturas de los poderes tradicionales imposibilitando un acuerdo vinculante sobre el tema.

En los primeros días de la cop21, celebrada en 2015 en París, los gobiernos de Brasil, India, China y Sudáfrica reafirmaron -por medio de una declaración conjunta- el principio de la "diferenciación" asegurando que los países desarrollados son quienes deben asumir el grueso de los esfuerzos en la lucha contra el cambio climático. Los poderes tradicionales, como la Unión Europea, argumentaron que

72 Saibal Dasgupta, "Copenhagen conference: India, China plan joint exit”, The Times of India, 28 de noviembre de 2009, en http://timesofindia.indiatimes. com/india/Copenhagen-conference-India-China-plan-joint-exit/articleshow/5279771.cms 
esta diferenciación ha quedado desfasada, teniendo en cuenta que China y ahora también India se encuentran entre las cuatro economías más contaminantes. ${ }^{73}$ De modo tal que se presentó un esquema de negociación internacional, en el que los poderes emergentes se vieron obligados a dejar de exhortar tan sólo por sus "derechos" en la esfera global, para asumir las "responsabilidades" que conlleva un papel crecientemente destacado en el ámbito mundial. Todo ello, con el explícito reconocimiento de su condición por parte de los poderes tradicionales, que ahora exigen un desempeño a la altura de las circunstancias.

Sorprendentemente, la Cumbre de París logró cerrar un acuerdo histórico por el cual todos los países firmantes se comprometieron a limitar sus emisiones. De este modo, se dejó de lado la disputa sobre la diferenciación abarcando a todos los signatarios. El texto estableció, empero, mayores exigencias para los países desarrollados quienes deberán "seguir encabezando los esfuerzos". ${ }^{74}$

En este sentido, China logró imponer sus posiciones, dado que el documento final establece que los compromisos de reducción de emisiones serán revisados cada cinco años. Sin embargo, incluye una excepción para aquellos que ya se hayan comprometido con recortes hasta el año 2030 (entre los cuales se encuentra Beijing). Pese a esto, la actitud del gobierno chino ha sido muy diferente de la que mostró en 2009, en Copenhague. En ese entonces, "China se encontraba en plena aplicación de un modelo que primaba el crecimiento por encima de cualquier cosa, incluidas las preocupaciones medioambientales. Hoy ese modelo está agotado y su población protesta de modo cada vez más contundente por el deterioro ecológico". ${ }^{75}$ Consecuentemente, el gobierno

73 "China e India rechazan un pacto del clima que les iguale a los países ricos", El País, sección internacional, 9 de diciembre de 2015, en http://internacional.elpais.com/internacional/2015/12/08/actualidad/1449585117_634927.html

74 "La Cumbre de París cierra un acuerdo histórico contra el cambio climático”, El País, sección internacional, 14 de diciembre de 2015, en http://internacional.elpais.com/internacional/2015/12/12/actualidad/1449910910_209267.html

75 "China defiende sus líneas rojas, pero por primera vez asume compromisos”, El País, sección internacional, 13 de diciembre de 2015, en http:/ /internacional.elpais.com/internacional/2015/12/12/actualidad/1449952042_129498.html 
chino ha prometido recortar sus emisiones por unidad de PIB entre un 60 y un $65 \%$ para 2030, con respecto de los niveles de 2005. Asimismo, aseguró que aumentará al $20 \%$ el porcentaje de combustibles no fósiles (renovables y energía nuclear) en su cesta de consumo energético (hoy representa el 11\%). ${ }^{76}$ Es importante apuntar que las conversaciones bilaterales establecidas entre el presidente de los Ee.uU., Barack Obama, y el chino, Xi Jinping, fueron uno de los factores determinantes para el éxito de este proceso negociador, al posibilitar un acuerdo entre las dos actuales economías más importantes y contaminantes del mundo.

\section{CONSIDERACIONES FINALES}

El análisis del papel desempeñado por los poderes medios emergentes en distintos esquemas decisorios internacionales demuestra que su actuación está produciendo ciertas modificaciones, pequeñas, progresivas, pero constantes de la estructura de gobernanza mundial. Como se sostuvo en la hipótesis de trabajo aquí propuesta, se observa que por medio de la ejecución de políticas exteriores proactivas y de alto perfil internacional, los gobiernos de estos poderes en ascenso se han involucrado crecientemente en múltiples ámbitos de deliberación global.

Desde el punto de vista de los poderes medios emergentes, India, Brasil y Sudáfrica han venido construyendo un largo camino que, a partir del nuevo siglo, tiene sus inicios en IBsa y continúa posteriormente en el marco de BRICs. Pese a los sucesivos cambios de gobiernos en cada uno de ellos (con los consecuentes cambios de estilo y/o de posicionamiento ideológico), la voluntad política de llevar adelante una mayor participación en las esferas globales se ha mantenido intacta. En este sentido, por lo que hace al discurso, han reivindicado su pertenencia al Sur, exhortando por ámbitos multilaterales que contemplen la lucha contra la pobreza y la desigualdad. Del mismo modo, han abogado por la defensa de los valores democráticos, la multietnicidad y la valorización 
de la figura del Estado, con un claro apoyo al respeto de la soberanía.

En el caso de China, también ha experimentado un ascenso internacional, que la coloca en el grupo de los países emergentes. Sin embargo, el gobierno del Gigante asiático ha cultivado un bajo perfil, con el propósito de convencer de que su emergencia político-económica no constituye una amenaza para el resto del sistema mundial.

En cuanto a Rusia, se observa que, pese a su pertenencia al BRICS, la realidad de la otrora superpotencia socialista de la Guerra Fría dista, no poco, de la situación de sus restantes compañeros de sigla. Tomando sólo en consideración las esferas multilaterales aquí planteadas, se advierte que recientemente, en 2012, logró ingresar a la OMC, por lo que sus condiciones en tal organismo son diferentes a las del resto de los emergentes. Con respecto de las esferas financieras, cabe recordar que el G-7 se convirtió en G-8 ${ }^{77}$ con su incorporación al finalizar la Guerra Fría, es decir ya participaba en estas reuniones de los poderes centrales. En cuanto al cambio climático, Rusia ya se encuentra incorporada en la categoría de países desarrollados, por lo que la reciente polémica de los emergentes no la involucra. Por todas estas razones, se distingue a Rusia del resto de los poderes emergentes, ya que su verdadera situación se asocia en mayor medida con un proceso político que busca su "reemergencia" en el orden internacional.

El creciente protagonismo de los poderes medios emergentes ha implicado mayores niveles de negociación con las grandes potencias tradicionales. En este sentido, la estrategia tanto de Estados Unidos, como de los restantes miembros del G-7, ha sido una política de creciente incorporación de los poderes emergentes en los ámbitos de decisión multilaterales, con el propósito de poder influir e incluso condicionar sus comportamientos, para que sus acciones futuras sean más predecibles y calculables. En efecto, los poderes tradicionales posibilitaron la incorporación de los emergentes en el G-20 financiero, paralizaron las conversaciones en la

${ }^{77}$ El G-7 decidió excluir a Rusia en marzo de 2014 como consecuencia de la anexión de Crimea por parte del gobierno de Putin. 
OMC ante posiciones contrapuestas -aunque continuaron negociando por fuera de esta institución- y exigieron una mayor responsabilidad de los poderes en ascenso con respecto del cambio climático. De modo tal que buscan impedir además la conformación de estructuras de poder alternativas a las ya existentes.

A la luz de lo hasta ahora expuesto, es posible asegurar que los asuntos mundiales están siendo manejados crecientemente bajo una lógica colectiva, en la cual participan poderes tradicionales, emergentes y reemergentes. Los cambios acontecidos en los últimos veinticinco años han delineado progresivamente un nuevo escenario que difícilmente tendrá marcha atrás. Es de advertir que el paulatino ascenso de estos nuevos poderes no tiene como correlato una caída del poderío norteamericano. Antes bien, se trata del nacimiento de una estructura de poder más compleja, donde los canales de negociación entre los poderes tradicionales, los emergentes y los reemergentes, son más frecuentes, necesarios e inevitables.

Asimismo, la actual dinámica de relaciones entre viejos y nuevos poderes se caracteriza por relaciones predominantemente pacíficas, pero con mayores grados de informalidad y pragmatismo. Como prueba de ello es posible observar que se negocia por dentro o por fuera de las históricas organizaciones internacionales -Naciones Unidas, Banco Mundial, FMI, OMC-, según las circunstancias de deliberación. Esta dinámica implica además el ejercicio de una cooperación limitada en el tiempo y concentrada en asuntos específicos -ya sean comerciales, financieros, sociales o medioambientales, entre otros. Lo cierto es que los temas son de naturaleza interméstica, se yuxtaponen y se vinculan entre sí, en los distintos ambientes de discusión global. Las alianzas, por tanto, son transitorias, cambiantes y dependen del espacio de negociación que se tome en consideración. En este sentido, las relaciones entre Estados Unidos y China resultan ilustrativas. Según el caso, ambas potencias se muestran dispuestas a alinearse, pactar informalmente o distanciarse. La táctica radica en nunca "dar jaque mate al rey". ${ }^{78}$

Resulta importante señalar que las transformaciones a las que aquí se hace referencia de ninguna manera implican un cambio

${ }^{78}$ Miguel Otero Iglesias, apud A. González, art. cit. 
del sistema económico e ideológico imperante, esto es del capitalismo internacional. La verdadera motivación de los nuevos emergentes radica en ingresar plenamente al sistema capitalista con mayores cuotas de poder, que, en todo caso, los habilite a cambiar o modificar las reglas internacionales según sus necesidades e intereses.

Los nuevos poderes pretenden ingresar al "club de los grandes" y constreñir cualquier posible comportamiento unilateral de Estados Unidos con arreglos multilaterales que, en principio, parecerían aspirar al establecimiento de un proceso decisorio con rasgos propios de un nuevo equilibrio del poder mundial. En otros términos, las actuales vinculaciones entre poderes tradicionales, emergentes y re-emergentes están dando lugar a un modus operandi caracterizado por relaciones ambiguas y verdaderamente pragmáticas.

\section{BibLIOGRAFÍA}

Amorim, Celso, "Brazilian Foreign Policy under president Lula (20032010): an overview", Revista Brasileira de Política Internacional, vol. 53, edición especial, 2010, pp. 214-240.

Amsden, Alice, A ascensao do “resto", São Paulo, Ed. Unesp, 2001.

Banco Mundial, "El Banco Mundial introduce una reforma de los derechos de voto y obtiene un aumento por valor de US $\$ 86.000$ millones", comunicado de prensa, sitio web oficial, 25 de abril de 2010.

Barbé, Esther, Relaciones internacionales, Madrid, Tecnos, 1995.

Behringer, Ronald, "Middle power leadership on human security", paper presented at the annual meeting of the Canadian Political Science Association, Nova Scotia, 2003, en http://www.cpsa-acsp.ca/paper-2003/behringer.pdf

BRICS, Declaración conjunta II Cumbre, 2010, pp. 2-3, en http://www. pravasitoday.com/wp-content/uploads/2011/04/BRICS-SUMMIT-2011-JOINT-DECLARATION1.pdf

BRICS, Declaración conjunta VI Cumbre, 2014, en http://www.mea.gov. in/bilateral-documents-es.htm?dtl/23635/Sixth+BRICS+Summit++Fortaleza+Declaration

Central Intelligence Agency, The World Factbook, en https://www.cia.gov/ library/publications/the-world-factbook/ 
Chapnick, Adam, "The middle power", Canadian Foreign Policy, vol. 7, núm. 2, 1999, pp. 73-82.

"China defiende sus líneas rojas, pero por primera vez asume compromisos", El País, sección internacional, 13 de diciembre de 2015, en http://internacional.elpais.com/internacional/2015/12/12/actualidad/1449952042_129498.html

"China e India rechazan un pacto del clima que les iguale a los países ricos”, El País, sección internacional, 9 de diciembre de 2015, en http://internacional.elpais.com/internacional/2015/12/08/actualidad/1449585117_634927.html

“¿Cómo logró India convertirse en la nueva China de la economía mundial?, BBC Mundo, 9 de febrero de 2016, en http://www.bbc.com/ mundo/noticias/2016/02/160208_economia_india_nueva_china_lf

Convención Marco de Naciones Unidas sobre Cambio Climático, sitio web oficial: https:/unfccc.int/files/essential_background/background_publications_htmlpdf/application/pdf/convsp.pdf

Cooper, Andrew, "The evolution of multilateralism in an intermediate state: the re-orientation of Canadian strategy in the economic and security arenas", en Paths to Power; Foreign Policy Strategies of Brazil, Canada, India and Mexico, Working Paper, Woodrow Wilson International Center, 13 de mayo de 1999.

- y Agata Antkeiwicz (eds.), Emerging Powers and Global Governance, Waterloo, Wilfried Laurier University Press, 2008.

—_, Richard Higgott y Kim Nossal, Relocating Middle Powers: Australia and Canada in a Changing World Order, Vancouver, ubc Press, 1993.

Cooper, David, "Somewhere Between Great and Small: Disentangling the Conceptual Jumble of Middle, Regional, and «Niche» Powers", The Journal of Diplomacy and International Relations, vol. 14, 2013, pp. 23-35.

Costa Vaz, Alcides (ed.), Intermediate status, regional leadership and security: India, Brazil and South Africa, Brasilia, Editora Universidade de Brasilia, 2006.

Cox, Robert, "Multilateralism and World Order", Review of International Studies, vol. 18, núm. 2, 1992, pp. 161-180.

Dadush, Uri, "La política comercial fortuita", Política Exterior, núm. 153, mayo-junio de 2013, en http://www.politicaexterior.com/articulos/ politica-exterior/la-politica-comercial-fortuita/ 
Dasgupta, Saibal, "Copenhagen conference: India, China plan joint exit", The Times of India, 28 de noviembre de 2009, en http:/ / timesofindia. indiatimes.com/india/Copenhagen-conference-India-China-plan-joint-exit/articleshow/5279771.cms

Derghougassian, Khatchik, "IBSA no gubernamental: movilización social, diplomacia ciudadana y gobernabilidad de seguridad en la integración Sur-Sur", en Juan Tokatlian (comp.), India, Brasil y Sudáfrica: el impacto de las nuevas potencias regionales, Buenos Aires, Libros del Zorzal, 2007, pp. 191-238.

Dewitt, David, "Los poderes medios y la seguridad regional”, en Juan Tokatlian (comp.), India, Brasil y Sudáfrica: el impacto de las nuevas potencias regionales, Buenos Aires, Libros del Zorzal, 2007, pp. 55-96.

Drezner, Daniel, "El nuevo orden mundial nuevo", Foreign Affairs: Latinoamérica, vol. 7, núm. 3, pp. 153-163.

"Emerging Economies", The Economist, 15 de noviembre de 2007.

Fagundes Visentini, Paulo, "O Brasil como Potência Média: Possibilidades de ação internacional”, en Ricardo Sennes (org.), O Brasil e a Política Internacional, São Paulo, IDESP, 1998.

FMI: Fondo Monetario Internacional, "Cómo toma sus decisiones el FMI", ficha técnica, sitio web oficial, 7 de abril de 2016, en https://www. imf.org/external/np/exr/facts/spa/governs.htm

—- "Las cuotas en el FMI", ficha técnica, sitio web oficial, 27 de enero de 2016, en https://www.imf.org/external/np/exr/facts/spa/quo tass.htm

Garten, Jeffrey, The Big Ten: The Big Emerging Markets and How They Will Change Our Lives, Nueva York, Basic Books, 1997.

Giaccaglia, Clarisa, "Condicionantes sociales en el proceso de formación de potencias mundiales. Un análisis de los países de IBSA a partir de un recorrido histórico", Relaciones Internacionales, núm. 15, octubre de 2010, pp. 33-60.

-, "El rol de India, Brasil y Sudáfrica en el sistema internacional contemporáneo: ¿qué tipo de desarrollo y para quién?”, Revista Ciência Ẽ Trópico, vol. 35, núm. 2, 2011, pp. 367-415.

— Estrategias de política exterior de los poderes medios emergentes en el escenario internacional de la post-Guerra Fría. El caso de IBSA-India, Brasil y Sudáfrica (2003-2010), tesis, Rosario, Universidad Nacional de Rosario, Argentina, 2012. 
González Aguayo, Leopoldo, "Aproximación a una teoría de las potencias medianas”, Relaciones Internacionales, vol. 3, núm. 8, 1975.

González, Alicia, "Los Brics y la gobernanza económica mundial", Política Exterior, núm. 164, marzo-abril de 2015, en http://www.politicaexterior.com/articulos/politica-exterior/los-brics-y-la-gobernanza-economica-mundial/

González, Guadalupe, "Incertidumbres de una potencia media regional: las nuevas dimensiones de la política exterior mexicana", en Olga Pellicer (comp.), La política exterior en México: desafíos en los ochenta, México, CIDE, colección Estudios Políticos, enero de 1983.

Grevi, Giovanni, "El mundo interpolar", Foreign Policy en español, abrilmayo de 2010, en http:/ / www.fp-es.org/el-mundo-interpolar

Haas, Richard, "La era de la no polaridad. Lo que seguirá al dominio de Estados Unidos”, Foreign Affairs: Lationamérica, vol. 87, núm 3, 2008, pp. 66-78.

Holbraad, Carsten, Las potencias medias en la política internacional, México, Fondo de Cultura Económica, 1989.

Huntington, Samuel, "The Lonely Superpower", Foreign Affairs, vol. 78, núm. 2, 1999, pp. 35-49.

Hurrell, Andrew "Brasil y la tormenta que se avecina", Foreign Affairs: Latinoamérica, vol. 9, núm. 2, 2009, pp. 43-54.

- y Sandeep Sengupta, "Emerging powers, North-South relations and global climate politics", International Affairs, vol. 88, núm. 3, 2012, pp. 463-484.

, "Hegemonía, liberalismo e ordem global: qual é o espaço para potencias emergentes?”, en Amrita Narlikar, A. Hurrell, M. Regina Soares De Lima, Monica Hirst, Neil Macfarlane y Rosemary Foot (comps.), Os bRICS e a Ordem Global, Río de Janeiro, FGV Editora, 2009, pp. 9-41.

, "Some Reflections on the Role of Intermediate Powers in International Institutions", Working Paper 244, "Paths to Power: Foreign Policy Strategies of Intermediate States", Washington, DC, Latin American Program, Woodrow Wilson International Centre, 2000, en http:/ /www.corteidh.or.cr/tablas/15011.PDF

Ikenberry, John, Michael Mastanduno y William C. Wohlforth, "Introduction. Unipolarity, State Behavior and Systemic Consequences", World Politics, vol. 61, núm. 1, 2009, pp. 1-27. 
Insulza, José Miguel, "El dilema de una potencia intermedia”, Nexos, núm. 102, 1 de junio de 1986, en http:/ / www.nexos.com.mx/?p=4630

Keet, Dot, Alternativas estratégicas Sur-Sur al sistema económico y régimen de poder globales, Ámsterdam, Trasnational Institute (TNI), 2006.

Khana, Parag, El segundo mundo: Imperios e influencias en el nuevo orden global, Nueva York, Random House, 2008.

Kornegay, Francis y Narnia Bohler Muller, Laying the BRICS of a New Global Order: From Yekaterinburg 2009 to Thekwini 2013, Sudáfrica, Africa Institute of South Africa, 2013.

Krauthamer, Charles, "The Unipolar Moment Revisited", The National Interest, invierno de 2002-2003, pp. 5-17.

Kugler, Jacek y A. F. K. Organski, "The Power Transition: A Retrospective and Prospective Evaluation”, en Manus I. Midlarski (ed.), Handbook of War Studies, Boston, Unwin Hyman, 2006, pp. 171-194.

"La Cumbre de París cierra un acuerdo histórico contra el cambio climático”, El País, sección internacional, 14 de diciembre de 2015, en http:/ /internacional.elpais.com/internacional/2015/12/12/actualidad/1449910910_209267.html

Lechini, Gladys, "Sudáfrica: la identidad nacional como construcción y el liderazgo regional como destino manifiesto" en Anabella Busso (comp.), Fuerzas profundas e identidad. Reflexiones en torno a su impacto sobre la política exterior. Un recorrido de Casos, Rosario, UNR editora, 2010, pp. 175-188.

"Los BRICS quieren ser el motor del desarrollo", La Nación, Buenos Aires, 15 de julio de 2014, en http://www.lanacion.com.ar/1710053-losbrics-quieren-ser-el-motor-del-desarrollo

Mackay, Robert, "The Canadian Doctrine of the Middle Powers", en Harvey Dyck y Peter Krosby (eds.), Empire and nations. Essays in honour of Frederic Soward, Toronto, University of Toronto Press, 1969.

Nolte, Detlef, "How to compare regional powers: analytical concepts and research topics”, Review of International Studies, vol. 36, núm. 4, 2010, pp. 881-901.

, "Potencias regionales en la política internacional: conceptos y enfoques de análisis", German Institute of Global and Area Studies (GIGA), núm. 30, octubre de 2006, en http:/ / www.giga-hamburg.de/ content/publikationen/pdf/wp30_nolte.pdf 
O’Neill, Jim, "Building Better Global Economic BRICs”, GS Global Economics, Paper 66, octubre de 2001, en http://www.goldman sachs. com/our-thinking/archive/archive-pdfs/build-better-brics.pdf , "Dreaming with BRICs: The Path to 2050", GS Global Economics, Paper 99, noviembre de 2003, en http://www.goldmansachs. com/our-thinking/archive/archive-pdfs/brics-dream.pdf

Orgaz, Luis, Luis Molina y Carmen Carrasco, "El creciente peso de las economías emergentes en la economía y gobernanza mundiales. Los países BRIC", Documentos Ocasionales, núm. 1101, Banco de España, 28 de marzo de 2011.

Palou, Jordi, "El concepto de potencia media. Los casos de España y México”, Revista CIDOB d'Afers Internacionals, núm. 26, 1993, pp. 7-35.

Pant, Harsh V., "The BRICs fallacy", The Washington Quarterly, vol. 36, núm. 3, 2013, pp. 91-105.

Pérez Llana, Carlos, “¿Potencias intermedias o países mayores? La política exterior de la Argentina, el Brasil y México”, en Luciano Tomassini (comp.), Relaciones Internacionales de la América Latina, México, Fondo de Cultura Económica, 1981.

"Potencias emergentes: China, India, Brasil y Sudáfrica", Vanguardia Dossier, núm. 12, 2004.

Rocha, Alberto y Daniel Morales, "El sistema político internacional de pos-Guerra Fría y el rol de las potencias regionales mediadoras", Espiral. Estudios de Estado y Sociedad, vol. 15, núm. 43, 2008, pp. 23-75.

Rosas, María Cristina, "El estudio de las potencias medias en las Relaciones Internacionales: los casos de Australia y Canadá”, Relaciones Internacionales, núm. 78, 1998.

Sahni, Varun, “¿Ancla flotante o plataforma de lanzamiento? Dinámica regional de los poderes emergentes" en Juan Tokatlian (comp.), India, Brasil y Sudáfrica: el impacto de las nuevas potencias regionales, Buenos Aires, Libros del Zorzal, 2007, pp. 97-126.

Sanahuja, José Antonio, "El G-20 y la Gobernanza Económica Global: cuestiones de Representatividad, Legitimidad y Eficacia”, Anuario Sociolaboral, núm. 2, Fundación 1 de mayo, 2012, pp. 197-213.

Sennes, Ricardo, "Países intermediários e fóruns multilaterais: algumas considerações", trabajo presentado en el $3^{\circ}$ Encontro Nacional da ABCP, Associação Brasileira de Ciência Política, Niteroi, 28 a 31 de julio de 2002. 
Sharma, Ruchir, "The broken BRICs", Foreign Affairs, noviembre-diciembre de 2012, en https://www.foreignaffairs.com/articles/brazil/2012-1022/broken-brics

Soares De Lima, María Regina y Mónica Hirst, "Brasil como país intermediário e poder regional”, en Amrita Narlikar, A. Hurrell, M. Regina Soares De Lima, Monica Hirst, Neil Macfarlane y Rosemary Foot (comps.), Os BRICs e a Ordem Global, Río de Janeiro, FGV Editora, 2009.

Talley, Ian, "Los BRICs pasan del brillo a las sombras", The Wall Street Journal Americas, reproducido en el diario La Nación, 20 de enero de 2016, en http:/ / www.lanacion.com.ar/1863647-los-brics-pasan-del-brillo-a-lassombras

The Boston Consulting Group, "The New Global Challenges. How 100 Top Companies from Rapidly Developing Economies are Changing the World", BCG Report, Boston, 2006.

"The great deceleration", The Economist, 27 de julio de 2013.

"The New Titans", The Economist, 14 de septiembre de 2006.

Viola, Eduardo, Eduardo Viola, "Climate governance in an international system under conservative hegemony: the role of major powers" $R \boldsymbol{e}$ vista Brasileira de Política Internacional, vol. 55, núm. especial, 2012, pp. 9-29.

Vogt, Susanna (coord.), Growth and Responsibility. The Positioning of emerging powers in the global governance system, Sankt Augustin-Berlín, Konrad-Adenauer-Stiftung, 2009.

Zakaria, Fareed, The post-American world, Nueva York, Norton, 2009.

Zelicovich, Julieta, "Signos de cambio en el Régimen Multilateral de Comercio: análisis de la elección de Roberto Carvalho de Azevêdo como Director General de la oMc”, Boletim Mundorama, 2013. 